Research Article

\title{
Robust Multisensor MeMBer Filter for Multiple Extended-Target Tracking
}

\author{
Xiaoke Lu, ${ }^{1}$ Zhiguo Zhang $\mathbb{D}^{2},{ }^{2}$ Qing Li $\mathbb{D}^{3},{ }^{3}$ and Jinping Sun ${ }^{2}$ \\ ${ }^{1}$ Nanjing Research Institute of Electronics Technology, Nanjing 210039, China \\ ${ }^{2}$ School of Electronics \& Information Engineering, Beihang University, Beijing 100191, China \\ ${ }^{3}$ Department of Engineering, University of Cambridge, Cambridge CB2 1PZ, UK \\ Correspondence should be addressed to Qing Li; q1289@cam.ac.uk \\ Received 3 April 2021; Revised 26 April 2021; Accepted 8 May 2021; Published 20 May 2021 \\ Academic Editor: Xiao Chen \\ Copyright (C) 2021 Xiaoke Lu et al. This is an open access article distributed under the Creative Commons Attribution License, \\ which permits unrestricted use, distribution, and reproduction in any medium, provided the original work is properly cited. \\ This paper develops a robust extended-target multisensor multitarget multi-Bernoulli (ET-MS-MeMBer) filter for enhancing the \\ unsatisfactory quality of measurement partitions arising in the classical ET-MS-MeMBer filter due to increased clutter intensities. \\ Specifically, the proposed method considers the influence of the clutter measurement set by introducing the ratio of the target \\ likelihood to the clutter likelihood. With the constraint of the clutter measurement set, it can obtain better multisensor measurement \\ partitioning results under the original two-step greedy partitioning mechanism. Subsequently, the single-target multisensor like- \\ lihood function for the clutter case is derived. Simulation results reveal a favorable comparison to the ET-MS-MeMBer filter in terms \\ of accuracy in estimating the target cardinality and target state under conditions with increased clutter intensities.
}

\section{Introduction}

Multiple-target tracking (MTT) [1] estimates the number and states of moving targets based on the sensor observations. Traditional MTT algorithms adopt a point target observation model [2], which assumes that one target generates at most one measurement per time step. However, in the high-resolution sensor system, one target may occupy multiple-resolution cells and, thus, can produce multiple measurements. Such targets are known as extended targets [3]. The measurements generated by the extended target provide information not only about the target's motion state but also the target's shape. This shape information, e.g., the size and structure of the extended target, can further facilitate the target classification and recognition task [4].

The conventional multiple-target tracking algorithm involving a data association step [5] has been widely used in the fields of medical image processing [6], network defense [7], and battlefield environment monitoring [8]. However, for extended-target tracking, the association between measurements and targets becomes more complicated and suffers from a "combinatorial explosion" problem.
Therefore, the random finite set (RFS) $[9,10]$ has received much attention in the MTT domain for the merit of avoiding explicit data association steps. This theory leads to the development of various MTT algorithms, including the probability hypothesis density (PHD) [11], cardinalized PHD (CPHD) [12], arithmetic average multi-Bernoulli (AA$\mathrm{MB}$ ) [13], and multisensor multitarget multi-Bernoulli (MSMeMBer) [14] filters.

The abovementioned filters based on the RFS have further been applied to the field of extended-target tracking [15-23]. Based on the PHD filter, an extended-target PHD (ET-PHD) filter was proposed in [15], and a Gaussian implementation of it was given in [16]. As the ET-PHD filter can only estimate the centroid state of the extended target, the PHD filters with shape estimation were proposed in $[17,18]$. In [17], a Gaussian inverse Wishart PHD (GIWPHD) filter was proposed, where the target kinematical state is modeled as a Gaussian distribution and the target extension is assumed to follow an inverse Wishart distribution. As an improvement to the GIW-PHD filter, the GGIW-PHD filter proposed in [18] can additionally obtain the estimation of target measurement rates. Besides the PHD filter, other 
filters based on the RFS [19-23] have also been modified to track the extended target. In [19], an extended-target CPHD filter that can estimate the centroid state of extended targets was proposed. Compared with the ET-PHD filter, this filter achieves better filtering results at the cost of higher computational complexity. Based on the labeled RFS, the Gamma Gaussian inverse Wishart (GGIW) implementation of the labeled multi-Bernoulli (LMB) filter was introduced in [20]. In [21-23], the Poisson multi-Bernoulli Mixture (PMBM) filter was generalized to the extended-target tracking case.

For the multisensor case, the extended-target MSMeMBer (ET-MS-MeMBer) filter and its Gaussian inverse Wishart (GIW) mixture implementation was proposed in [24]. The ET-MS-MeMBer filter assumes that the observation of extended targets obeys the approximate Poisson-Body (APB) model [25], and the update process of the MS-MeMBer filter is modified accordingly. However, this method ignores the constraint of the clutter measurement set in the multisensor measurement partitioning process, which may degenerate the quality of the multisensor measurement partition and, consequently, the filtering performance of the MS-MeMBer filter in high-clutter-density scenarios. To solve this problem, a robust ET-MS-MeMBer filter is proposed in this paper. By introducing the ratio of the target likelihood to the clutter likelihood, the influence of the clutter measurement set is considered. Therefore, better measurement partitions can be obtained under the original two-step partitioning mechanism [14]. The single-target multisensor likelihood function for the clutter case is also derived. Simulation results show that compared to the ETMS-MeMBer filter, the proposed filter has higher accuracy in estimating the target cardinality and target state under high-clutter-density conditions.

The rest of this paper is organized as follows. Section 2 gives a review of the ET-MS-MeMBer filter. The proposed filter is derived in Section 3. The simulation results are presented in Section 4, and the conclusions are given in Section 5.

\section{The ET-MS-MeMBer Filter}

2.1. The Prediction Step. Let $\mathrm{x}$ and $X=\left\{x_{1}, \ldots, x_{M_{k-1 \mid k-1}}\right\}$ denote a single-target state and a multitarget state, where $M_{k-1 \mid k-1}$ is the cardinality of targets at time $k-1$. Assume that, at time $k-1$, the multitarget posterior density is a multi-Bernoulli

$$
\pi_{k-1 \mid k-1}(X)=\left\{\left(r_{k-1 \mid k-1}^{(i)}, p_{k-1 \mid k-1}^{(i)}(x)\right)\right\}_{i=1}^{M_{k-1 \mid k-1}}
$$

where each $\left(r_{k-1 \mid k-1}^{(i)}, p_{k-1 \mid k-1}^{(i)}(x)\right)$ represents an independent Bernoulli density with probability $r_{k-1 \mid k-1}^{(i)}$ and distribution $p_{k-1 \mid k-1}^{(i)}(x)$.

Then, the predicted multitarget density is also a multiBernoulli

$$
\begin{aligned}
\pi_{k \mid k-1}(X)= & \left\{\left(r_{s, k \mid k-1}^{(i)}, p_{s, k \mid k-1}^{(i)}(x)\right)\right\}_{i=1}^{M_{k-1 \mid k-1}} \\
& \cup\left\{\left(r_{b, k}^{(j)}, p_{b, k}^{(j)}(x)\right)\right\}_{j=1}^{M_{b, k}},
\end{aligned}
$$

where $\left(r_{b, k}^{(j)}, p_{b, k}^{(j)}(\mathrm{x})\right)$ and $M_{b, k}$ are the Bernoulli density and the cardinality of newborn targets at time $k$. The survival Bernoulli density $\left(r_{s, k \mid k-1}^{(i)}, p_{s, k \mid k-1}^{(i)}(x)\right)$ follows

$$
\begin{aligned}
r_{s, k \mid k-1}^{(i)} & =r_{k-1 \mid k-1}^{(i)}\left\langle p_{k-1 \mid k-1}^{(i)}, \rho_{\mathrm{sv}}\right\rangle, \\
p_{s, k \mid k-1}^{(i)}(x) & =\frac{\left\langle f_{k \mid k-1}(x \mid \cdot), \rho_{\mathrm{sv}} p_{k-1 \mid k-1}^{(i)}(x)\right\rangle}{\left\langle p_{k-1 \mid k-1}^{(i)}(x), \rho_{\mathrm{sv}}\right\rangle},
\end{aligned}
$$

where $\rho_{\mathrm{sv}}$ is the probability that a target can survive to the next time, $\langle\cdot, \cdot\rangle$ is the inner product function, and $f_{k \mid k-1}(\mathrm{x} \mid \cdot)$ is the state transition function.

\subsection{The Distance Partition and Multisensor Measurement} Partition. The update step of the ET-MS-MeMBer filter requires the multisensor measurement partitions. Since each extended target may generate multiple measurements at each scan, our first task is to divide each sensor's measurements into groups that contain measurements from the same target. Here, we utilize a distance partition method [17] which is based on the general belief that measurements from the same extended target are spatially close to each other.

Let $Z_{i}=\left\{z_{i}^{1}, \ldots, z_{i}^{n}\right\}$ be the collection of all measurements generated on sensor $i$ at time $k$, where $z_{i}^{l}(1 \leq l \leq n)$ is the $l$-th element in $Z_{i}$ and $n$ is the number of the measurements. The distance partition of $Z_{i}$ can be denoted as $\mathbf{D}_{i}=\left\{w_{i}^{0}, w_{i}^{1}, \ldots, w_{i}^{\left|\mathbf{D}_{i}\right|-1}\right\}$, where

$$
\begin{gathered}
w_{i}^{l} \cap w_{i}^{m}=\varnothing \quad\left(0 \leq l \neq m \leq\left|\mathbf{D}_{i}\right|-1\right), \\
w_{i}^{0} \cup w_{i}^{1} \cup \cdots \cup w_{i}^{\left|D_{i}\right|-1}=Z_{i} .
\end{gathered}
$$

$|\cdot|$ is the cardinality function. Each disjoint subset $w_{i}^{j}$ ( $j=0$ for the clutter measurement subset and $0<j \leq\left|\mathbf{D}_{i}\right|-1$ for the target measurement subset) in partition $\mathbf{D}_{i}$ contains several measurements in $Z_{i}$ and satisfies $\left|w_{i}^{j}\right| \geq 0 . \mathscr{D}_{i}$ is the collection of all possible distance partitions $\mathbf{D}_{i}$.

Multisensor measurement partition aims to group all sensors' measurements from the same target into the same subset. We consider a multisensor measurement set $Z_{1: S}=\left\{Z_{1}, \ldots, Z_{S}\right\}$, where $S$ is the number of sensors. Suppose that, at time $k$, the predicted multitarget density contains $M_{k \mid k-1}$ Bernoulli components. For a given set of distance partitions $\mathbf{D}_{1}, \ldots, \mathbf{D}_{S}$, the multisensor measurement partition can be defined as

$$
\begin{aligned}
& \mathbf{P}=\left\{W_{1: S}^{0}, W_{1: S}^{1}, \ldots, W_{1: S}^{M_{k \mid k-1}}\right\}, \text { where } \\
& W_{1: S}^{l}=\left\{w_{1}^{l_{1}}, \ldots, w_{S}^{l_{S}}\right\} \\
& \quad \cdot\left(1 \leq l \leq M_{k \mid k-1}, \text { and } w_{1}^{l_{1}} \in \mathbf{D}_{1}, \ldots, w_{1}^{l_{S}} \in \mathbf{D}_{S}\right), \\
& \quad W_{1: S}^{l} \cap W_{1: S}^{j} \quad\left(0 \leq l \neq j \leq M_{k \mid k-1}\right), \\
& \\
& W_{1: S}^{0} \cup W_{1: S}^{1} \cup \cdots \cup W_{1: S}^{M_{k \mid k-1}}=Z_{1: S} .
\end{aligned}
$$

Here, $W_{1: S}^{0}$ is the clutter measurement set generated by all $S$ sensors, and $W_{1: S}^{l}(l>0)$ is the target measurement set corresponding to the $l$-th Bernoulli component. $\mathscr{P}$ is defined 
as the collection of all possible multisensor measurement partitions $\mathbf{P}$. Finally, we define the mapping function $T_{W_{1: S}^{l} s}=\left\{\left(i, l_{i}\right) \mid w_{i}^{l_{i}} \in W_{1: S}^{l}\right\}$, where $\left(i, l_{i}\right)$ specifies the sensor label $i$ and the index $l_{i}$ of $w_{i}^{l_{i}}$ in $\mathbf{D}_{i}$.

2.3. The Update Step. Suppose that, at time $k$, the predict multitarget density is a multi-Bernoulli consisting of $M_{k \mid k-1}$ independent Bernoulli component:

$$
\pi_{k \mid k-1}(X)=\left\{\left(r_{k \mid k-1}^{(j)}, p_{k \mid k-1}^{(j)}(x)\right)\right\}_{j=1}^{M_{k \mid k-1}}
$$

Then, the posterior density can be approximated by a multi-Bernoulli as follows:

$$
\pi_{k \mid k}(X)=\bigcup_{\mathbf{P} \in \mathscr{P}} \bigcup_{j=1}^{M_{k \mid k-1}}\left\{\left(r_{k \mid k}^{(\mathbf{P}, j)}, p_{k \mid k}^{(\mathbf{P}, j)}(x)\right)\right\} .
$$

The updated probability $r_{k \mid k}^{(\mathbf{P}, j)}$ and the distribution $p_{k \mid k}^{(\mathbf{P}, j)}(x)$ follow

$$
\begin{aligned}
r_{k \mid k}^{(\mathbf{P}, j)} & = \begin{cases}\alpha_{P} \frac{r_{k \mid k-1}^{(j)}\left\langle p_{k \mid k-1}^{(j)}, \eta\right\rangle}{1-r_{k \mid k-1}^{(j)}+r_{k \mid k-1}^{(j)}\left\langle p_{k \mid k-1}^{(j)}, \eta\right\rangle}, & W_{1: S}^{j}=\varnothing, \\
\alpha_{\mathbf{P}}, & W_{1: S}^{j} \neq \varnothing,\end{cases} \\
p_{k \mid k}^{(\mathbf{P}, j)}(x) & = \begin{cases}\frac{p_{k \mid k-1}^{(j)}(x) \eta(x)}{\left\langle p_{k \mid k-1}^{(j)}, \eta\right\rangle}, & W_{1: S}^{j}=\varnothing, \\
\frac{p_{k \mid k-1}^{(j)}(x) f\left(W_{1: S}^{j} \mid x\right)}{\int p_{k \mid k-1}^{(j)}(x) f\left(W_{1: S}^{j} \mid x\right) \mathrm{d} x}, & W_{1: S}^{j} \neq \varnothing,\end{cases}
\end{aligned}
$$

where $\eta(x)$ is the probability that a target is not detected by any sensor and $f\left(W_{1: S}^{j} \mid x\right)$ is the single-target multisensor likelihood function. $\alpha_{\mathbf{P}}$ is the weight of $\mathbf{P}$ in $\mathscr{P}$, which can be calculated as the score of $\mathbf{P}$ divided by the sum of all partitioning scores in $\mathscr{P}$ :

$$
\alpha_{\mathbf{P}}=K_{\mathbf{P}} \frac{\prod_{j=1}^{M_{k \mid k-1}} \varphi_{W_{1: S}^{j}}^{j}[1]}{\sum_{\mathbf{M} \in \mathbf{P}} K_{\mathbf{M}} \prod_{j=1}^{M_{k \mid k-1}} \varphi_{W_{1: S}^{j}}^{j}[1]}
$$

where

$$
\begin{aligned}
\varphi_{W_{1: S}^{j}}^{j}[u] & = \begin{cases}1-r_{k \mid k-1}^{(j)}+r_{k \mid k-1}^{(j)}\left\langle p_{k \mid k-1}^{(j)}, u \eta\right\rangle, & W_{1: S}^{j}=\varnothing, \\
r_{k \mid k-1}^{(j)} \int u(x) p_{k \mid k-1}^{(j)}(x) f\left(W_{1: S}^{j} \mid x\right) \mathrm{d} x, & W_{1: S}^{j} \neq \varnothing,\end{cases} \\
K_{\mathbf{P}} & =\left[\prod_{i=1}^{S} C_{i}^{\left(\left|w_{i}^{0}\right|\right)}(0)\right] \times\left[\prod_{j=1}^{M_{k \mid k-1}} \prod_{\left(i, l_{i}\right) \in T_{W_{1: S}^{j}}}\left(\mid w_{i}^{\left.l_{i} \mid !\right)}\right] .\right.
\end{aligned}
$$

$K_{\mathbf{P}}$ and $\varphi_{W_{1: S}^{j}}^{j}$ [1] quantify how likely the set $W_{1: S}^{0}$ is a clutter set, and the set $W_{1: s}^{j}(j>0)$ is associated with the $j$-th Bernoulli component, respectively. $C_{i}^{(n)}(x)$ is the $n$-th derivative of the clutter probability generating function $C_{i}(x)=e^{\lambda_{i} x-\lambda_{i}}$, and $\lambda_{i}$ is the clutter intensity of sensor $i$.

\section{The Robust ET-MS-MeMBer Filter}

3.1. The Constraint of the Clutter Measurement Set. In [14], a two-step greedy partitioning mechanism is proposed, which can obtain several high-scoring measurement partitions. In this mechanism, the target measurement set corresponding to each Bernoulli component is first obtained separately, and then, the target measurement sets corresponding to different Bernoulli components are combined to obtain all possible measurement partitions. However, it can be seen from (9) that the two-step greedy partitioning mechanism ignores the influence of the clutter measurement set $W_{1: s}^{0}$. Considering the constraint of the clutter measurement set, $\alpha_{\mathbf{P}}$ can be rewritten as

$$
\begin{aligned}
\alpha_{\mathbf{P}} & =\frac{\left(K_{\mathbf{P}} / \prod_{i=1}^{S} \lambda_{i}^{\left|Z_{i}\right|} e^{-\lambda_{i}}\right) \prod_{j=1}^{M_{k \mid k-1}} \varphi_{W_{1: S}^{j}}^{j}[1]}{\sum_{\mathbf{M} \in \mathscr{P}}\left(K_{\mathbf{M}} / \prod_{i=1}^{S} \lambda_{i}^{\left|Z_{i}\right|} e^{-\lambda_{i}}\right) \prod_{j=1}^{M_{k \mid k-1}} \varphi_{W_{1: S}^{j}}^{j}[1]}, \\
& =\frac{\prod_{j=1}^{M_{k \mid k-1}}\left(\varphi_{W_{1: S}^{j}}^{j}[1] \cdot \prod_{\left(i, l_{i}\right) \in T_{W_{1: S}^{j}}}\left(\left|w_{i}^{l_{i}}\right| ! / \lambda_{i}^{\left|w_{i}^{l_{i}}\right|}\right)\right)}{\sum_{\mathbf{M} \in \mathscr{P}} \prod_{j=1}^{M_{k \mid k-1}}\left(\varphi_{W_{1: S}^{j}}^{j}[1] \cdot \prod_{\left(i, l_{i}\right) \in T_{W_{1: S}^{j}}}\left(\left|w_{i}^{l_{i} \mid}\right| ! / \lambda_{i}^{\left|w_{i}^{l_{i}}\right|}\right)\right)} .
\end{aligned}
$$


We introduce the function

$$
\widetilde{\varphi}_{W_{1: S}^{j}}^{j}[1]=\varphi_{W_{1: S}^{j}}^{j}[1] \cdot \prod_{\left(i, l_{i}\right) \in T_{W_{1: S}^{j}}} \frac{\left|w_{i}^{l_{i} \mid}\right| !}{\mid w_{i}^{l_{i} \mid}},= \begin{cases}1-r_{k \mid k-1}^{(j)}+r_{k \mid k-1}^{(j)}\left\langle p_{k \mid k-1}^{(j)}, u \eta\right\rangle, & W_{1: s}^{j}=\varnothing, \\ r_{k \mid k-1}^{(j)} \int u(x) p_{k \mid k-1}^{(j)}(x) f\left(W_{1: S}^{j} \mid x\right) \prod_{\left(i, l_{i}\right) \in T_{W_{1: S}^{j}}} \frac{\left|w_{i}^{l_{i} \mid}\right| !}{\left|w_{i}^{l_{i} \mid}\right|} \mathrm{d} x, \quad W_{1: S}^{j} \neq \varnothing,\end{cases}
$$

which denotes the ratio of the likelihood that $W_{1: S}^{j}$ is generated by a target to the likelihood that $W_{1: S}^{j}$ is from the clutter.
According to [24], the single-target multisensor likelihood function follows

$$
f\left(W_{1: S}^{j} \mid x\right)=\prod_{\left(i, l_{i}\right) \in T_{W_{1: S}^{j}}} \frac{p_{i, d} \gamma_{i}^{\left|w_{i}^{l_{i}}\right|} e^{-\gamma_{i}}}{\left|w_{i}^{l_{i}}\right| !} \prod_{z \in w_{i}^{l_{i}}} \frac{h_{i}(z \mid x)}{c_{i}(z)} \times \prod_{(i, *) \notin T_{W_{1: S}^{j}}}\left(1-p_{i, d}+p_{i, d} e^{-\gamma_{i}}\right),
$$

where $\gamma_{i}$ is the mean of the extended measurements, $p_{i, d}$ is the target detection probability by sensor $i, h_{i}(z \mid x)$ is the measurement likelihood of sensor $i$, and $c_{i}(z)$ is the clutter intensity.

Hence, (12) becomes

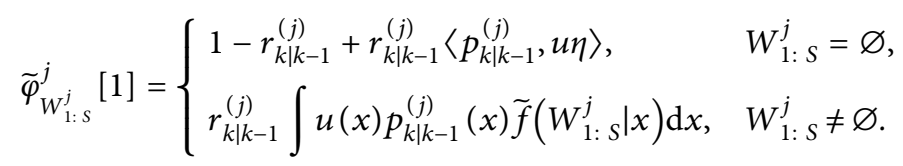

In (14), $\tilde{f}\left(W_{1: S}^{j} \mid x\right)$ denotes the single-target multisensor likelihood function for the clutter case and follows

$$
\tilde{f}\left(W_{1: S}^{j} \mid x\right)=\prod_{\left(i, l_{i}\right) \in T_{W_{1: S}^{j}}} p_{i, d} \gamma_{i}^{\left|w_{i}^{l_{i}}\right|} e^{-\gamma_{i}} \prod_{z \in w_{i}^{l_{i}}} \frac{h_{i}(z \mid x)}{\lambda_{i} c_{i}(z)} \times \prod_{\left(i,{ }^{*}\right) \notin T_{W_{1: S}^{j}}}\left(1-p_{i, d}+p_{i, d} e^{-\gamma_{i}}\right) .
$$

Finally, (11) becomes

$$
\alpha_{\mathbf{P}}=\frac{\prod_{j=1}^{M_{k \mid k-1}} \widetilde{\varphi}_{W_{1: S}^{j}}^{j}[1]}{\sum_{\mathbf{M} \in \mathbf{P}} \prod_{j=1}^{M_{k \mid k-1}} \widetilde{\varphi}_{W_{1: S}^{j}}^{j}[1]}
$$

It can be seen from (16) that the influence of the clutter measurement set has been introduced into the two-step greedy partitioning mechanism.
3.2. The Numerical Implementation. We define the target state as $x=[\tilde{x}, X]$, where $\tilde{x}=[p x, p y, v x, v y, a x, a y]^{T}$ contains the target position, target velocity, and target acceleration and $X$ represents the target shape. Utilizing the GIW mixture from $[17,24]$, we can derive a numerical implementation of the proposed filter.

Suppose that the distributions of the Bernoulli component at time $k-1$ and the newborn target at time $k k$ are both GIW mixtures as follows: 


$$
\begin{aligned}
p_{k-1 \mid k-1}^{(i)}(x) & =\sum_{n=1}^{J_{k-1 \mid k-1}^{(i)}} w_{n, k-1 \mid k-1}^{(i)} \mathcal{N}\left(\tilde{x} ; m_{n, k-1 \mid k-1}^{(i)}, P_{n, k-1 \mid k-1}^{(i)} \otimes X\right) \mathscr{I}\left(X ; v_{n, k-1 \mid k-1}^{(i)}, V_{n, k-1 \mid k-1}^{(i)}\right), \\
p_{b, k}^{(j)}(x) & =\sum_{n=1}^{J_{b, k}^{(j)}} w_{b, n, k}^{(j)} \mathcal{N}\left(\tilde{x} ; m_{b, n, k}^{(j)}, P_{b, n, k}^{(j)} \otimes X\right) \mathscr{I}\left(X ; v_{b, n, k}^{(j)}, V_{b, n, k}^{(j)}\right) .
\end{aligned}
$$

$J_{k-1 \mid k-1}^{(i)}$ and $J_{b, k}^{(j)}$ are the number of the GIW component. $\otimes$ denotes the Kronecker product. $w_{n, k-1 \mid k-1}^{(i)}$ and $w_{b, n, k}^{(j)}$ are the weight of the corresponding GIW component. $\mathcal{N}(\tilde{x} ; m, P)$ is a Gaussian function with mean $m$ and variance $P . \mathscr{I}(X ; v, V)$ is an inverse Wishart distribution with degrees of freedom $v$ and inverse scale matrix $V$.

Then, the distribution of the predicted Bernoulli component is also a GIW mixture, and the legacy Bernoulli component at time $k$ follows

$$
\begin{aligned}
r_{s, k \mid k-1}^{(i)} & =r_{k \mid k-1}^{(i)} \rho_{\mathrm{sv}}, \\
p_{s, k \mid k-1}^{(i)}(x) & =\sum_{n=1}^{J_{k-1 \mid k-1}^{(i)}} w_{n, k-1 \mid k-1}^{(i)} \mathcal{N}\left(\tilde{x} ; m_{n, k \mid k-1}^{(i)}, P_{n, k \mid k-1}^{(i)} \otimes X\right) \mathscr{I}\left(X ; v_{n, k \mid k-1}^{(i)}, V_{n, k \mid k-1}^{(i)}\right) .
\end{aligned}
$$
satisfy

In (19), the Gaussian and the inverse Wishart parameters

$$
\begin{aligned}
& m_{n, k \mid k-1}^{(i)}=\left(F_{k \mid k-1} \otimes \mathbf{I}_{d}\right) m_{n, k-1 \mid k-1}^{(i)}, \\
& P_{n, k \mid k-1}^{(i)}=F_{k \mid k-1} P_{n, k-1 \mid k-1}^{(i)} F_{k \mid k-1}^{T}+Q_{k \mid k-1}, \\
& v_{n, k \mid k-1}^{(i)}=v_{n, k-1 \mid k-1}^{(i)} e^{-\left(T_{s} / \tau\right)},
\end{aligned}
$$

$$
V_{n, k \mid k-1}^{(i)}=V_{n, k-1 \mid k-1}^{(i)} \frac{V_{n, k \mid k-1}^{(i)}-d-1}{V_{n, k-1 \mid k-1}^{(i)}-d-1}
$$

$$
F_{k \mid k-1}=\left[\begin{array}{ccc}
1 & T_{s} & (1 / 2) T_{s} \\
0 & 0 & T_{s} \\
0 & 0 & e^{\left(-T_{s} / \theta\right)}
\end{array}\right] \text { is the state transition matrix. }
$$

$Q_{k \mid k-1}$ is the process noise covariance matrix. $\tau$ is a temporal decay constant. $T_{s}$ is the sampling period. $\theta$ is the maneuver correlation time. $\mathbf{I}_{d}$ is the unit matrix of size $d$.

If the distribution of the predicted Bernoulli component satisfies

$$
p_{k \mid k-1}^{(i)}(x)=\sum_{n=1}^{J_{k \mid k-1}^{(i)}} w_{n, k \mid k-1}^{(i)} \mathcal{N}\left(\tilde{x} ; m_{n, k \mid k-1}^{(i)}, P_{n, k \mid k-1}^{(i)} \otimes X\right) \mathscr{I}\left(X ; v_{n, k \mid k-1}^{(i)}, V_{n, k \mid k-1}^{(i)}\right)
$$

then the updated Bernoulli density follows

$$
\begin{aligned}
& r_{k \mid k}^{(\mathbf{P}, j)}= \begin{cases}\alpha_{\mathbf{P}} \frac{r_{k \mid k-1}^{(j)} \prod_{i=1}^{S}\left(1-p_{i, d}+p_{i, d} e^{-\gamma_{i}}\right)}{1-r_{k \mid k-1}^{(j)}+r_{k \mid k-1}^{(j)} \prod_{i=1}^{S}\left(1-p_{i, d}+p_{i, d} e^{-\gamma_{i}}\right)}, & W_{1: S}^{j}=\varnothing, \\
\alpha_{\mathbf{P},} & W_{1: S}^{j} \neq \varnothing,\end{cases} \\
& p_{k \mid k}^{(\mathbf{P}, j)}(x)= \begin{cases}\sum_{n=1}^{J_{k \mid k-1}^{(j)}} w_{n, k \mid k-1}^{(j)} \mathcal{N}\left(\tilde{x} ; m_{n, k \mid k-1}^{(j)}, P_{n, k \mid k-1}^{(j)} \otimes X\right) \mathscr{I}\left(X ; v_{n, k \mid k-1}^{(j)}, V_{n, k \mid k-1}^{(j)}\right), & W_{1: S}^{j}=\varnothing, \\
\sum_{n=1}^{(j)} w_{n, k \mid k}^{(\mathbf{P}, j)} \mathcal{N}\left(\tilde{x} ; m_{n, k \mid k}^{(\mathbf{P}, j)}, P_{n, k \mid k}^{(\mathbf{P}, j)} \otimes X\right) \mathscr{I}\left(X ; v_{n, k \mid k}^{(\mathbf{P}, j)}, V_{n, k \mid k}^{(\mathbf{P}, j)}\right), & W_{1: S}^{j} \neq \varnothing .\end{cases}
\end{aligned}
$$


In (26), the weight of the GIW component follows

$$
w_{n, k \mid k}^{(\mathbf{P}, j)}=\frac{w_{n, k \mid k-1}^{(j)} \prod_{\left(i, l_{i}\right) \in T_{W_{1: S}^{j}}} p_{i, d} \gamma_{i}^{\left|w_{i}^{l_{i}}\right|} e^{-\gamma_{i}} q z\left(w_{i}^{l_{i}}\right) \prod_{z \in w_{i}^{l_{i}}}\left(1 / \lambda_{i} c_{i}(z)\right) \times \prod_{(i, *) \notin T_{W_{1: S}^{j}}}\left(1-p_{i, d}+p_{i, d} e^{-\gamma_{i}}\right)}{\sum_{m=1}^{J_{k \mid k-1}^{(j)}} w_{n, k \mid k-1}^{(j)} \prod_{\left(i, l_{i}\right) \in T_{W_{1: S}^{j}}} p_{i, d} \gamma_{i}^{\left|w_{i}^{l_{i}}\right|} e^{-\gamma_{i}} q z\left(w_{i}^{l_{i}}\right) \prod_{z \in w_{i}^{l_{i}}}\left(1 / \lambda_{i} c_{i}(z)\right) \times \prod_{(i, *) \notin T_{W_{1: S}^{j}}}\left(1-p_{i, d}+p_{i, d} e^{-\gamma_{i}}\right)},
$$

where $q z\left(w_{i}^{l_{i}}\right)$ is the likelihood ratio.

$$
\begin{aligned}
& \mathcal{N}\left(\tilde{x} ; m_{n, k \mid k}^{(\mathbf{P}, j)}, P_{n, k \mid k}^{(\mathbf{P}, j)} \otimes X\right) \cdot \mathscr{I}\left(X ; v_{n, k \mid k}^{(\mathbf{P}, j)}, V_{n, k \mid k}^{(\mathbf{P}, j)}\right) \\
& \propto \mathcal{N}\left(\tilde{x} ; m_{n, k \mid k-1}^{(j)}, P_{n, k \mid k-1}^{(j)} \otimes X\right) \cdot \mathscr{I}\left(X ; v_{n, k \mid k-1}^{(j)}, V_{n, k \mid k-1}^{(j)}\right) \cdot \prod_{\left(i, l_{i}\right) \in T_{w_{1: S}^{j}}} \prod_{z \in w_{i}^{l_{i}}} h_{i}(z \mid x),
\end{aligned}
$$

where

$$
\mathscr{N}(\tilde{x} ; m, P) \cdot \mathscr{I}(X ; v, V) \cdot \prod_{z \in w_{i}^{l_{i}}} h_{i}(z \mid x)=q z\left(w_{i}^{l_{i}}\right) \cdot \mathscr{N}\left(\tilde{x} ; m^{\prime}, P^{\prime}\right) \cdot \mathscr{I}\left(X ; v^{\prime}, V^{\prime}\right)
$$

In (28), the updated GIW parameters follow

$$
\text { where }
$$

$$
\begin{aligned}
m^{\prime} & =m+\left(K \otimes \mathbf{I}_{d}\right) \cdot\left(\widetilde{Z}_{k}^{W}-\left(H_{k} \otimes \mathbf{I}_{d}\right) m\right), \\
v^{\prime} & =v+\left|w_{i}^{l_{i}}\right|, \\
V^{\prime} & =V+N+Z_{k}^{W},
\end{aligned}
$$

$$
\begin{aligned}
\widetilde{Z}_{k}^{W} & =\frac{1}{\left|w_{i}^{l_{i}}\right|} \sum_{z \in w_{i}^{l_{i}}} z, \\
Z_{k}^{W} & =\sum_{z \in w_{i}^{l_{i}}}\left(z-\widetilde{Z}_{k}^{W}\right)\left(z-\widetilde{Z}_{k}^{W}\right)^{T}, \\
K & =P H_{k}^{T}\left(H_{k} P H_{k}^{T}+\frac{1}{\left|w_{i}^{l_{i}}\right|}\right)^{-1}, \\
N & =\left(H_{k} P H_{k}^{T}+\frac{1}{\left|w_{i}^{l_{i}}\right|}\right)^{-1}\left(\widetilde{Z}_{k}^{W}-\left(H_{k} \otimes \mathbf{I}_{d}\right) m\right)\left(\widetilde{Z}_{k}^{W}-\left(H_{k} \otimes \mathbf{I}_{d}\right) m\right)^{T} .
\end{aligned}
$$


Here, $H_{k}$ is the observation matrix at time $k$.

\section{Experimental Results}

In this section, we consider tracking 4 extended targets moving within an observation area of size $[-1000 \mathrm{~m}, 1000 \mathrm{~m}] \times$ $[-1000 \mathrm{~m}, 1000 \mathrm{~m}]$. The target shape is modeled as an ellipse [17] with a major axis $A=20$ and a minor axis $a=5$. The duration time is $80 \mathrm{~s}$. In the simulation, the constant acceleration (CA) model is considered. The target trajectories are shown in Figure 1. The initial position and the survival time of all the targets are shown in Table 1.

In (22), the sampling period $T_{s}=1 \mathrm{~s}$ and the temporal decay constant $\tau=5 \mathrm{~s}$. The maneuver correlation time $\theta=0.5$. In the two-step measurement partitioning method [14], the number of subsets in $\mathbf{P}$ is no more than $W_{\max }=6$, and the number of partitions in $\mathscr{P}$ is no more than $P_{\max }=6$. The pruning threshold is set as $T_{\max }=10^{-5}$, and the number of the GIW components of each Bernoulli density is up to $J_{\max }=100$. The merge threshold of Gaussian components is $U_{G}=50$, and the merge threshold of inverse Wishart components is $U_{\mathrm{IW}}=50$. We use the optimal subpattern assignment (OSPA) distance [26] as the metric to evaluate the filtering performance. The OSPA distance accounts for estimation errors in both position and cardinality, and in this section, we set the order $p=1$ and the penalty factor $c=100$.

Now, we consider observations from 4 sensors. The sensors are located at $[-900 \mathrm{~m}, 0 \mathrm{~m}],[0 \mathrm{~m},-900 \mathrm{~m}],[900 \mathrm{~m}$, $0 \mathrm{~m}]$, and $[0 \mathrm{~m}, 900 \mathrm{~m}]$, respectively. For every sensor, the detection probability $p_{i, d}=0.7$ and the clutter density parameter $\lambda_{i}=80$. The measurement noise standard deviations along $x-y$ coordinates of each sensor are $\varepsilon_{x_{1}}=\varepsilon_{y_{1}}=5 \mathrm{~m}$, $\varepsilon_{x_{2}}=\varepsilon_{y_{2}}=5 \mathrm{~m}, \varepsilon_{x_{3}}=\varepsilon_{y_{3}}=10 \mathrm{~m}$, and $\varepsilon_{x_{4}}=\varepsilon_{y_{4}}=10 \mathrm{~m}$. The mean of the extended measurements is set as $\gamma_{i}=20$. Figure 2 shows the measurements collected from sensor 1 . The estimated states by using the proposed method and the ETMS-MeMBer filter are shown in Figures 3 and 4, respectively. We can observe that compared with the ET-MSMeMBer filter, the proposed method can obtain more accurate state estimation results. We also compare the filtering performance of the ET-MS-MeMBer filter with that of the proposed method via 50 Monte Carlo runs. Figure 5 shows the cardinality estimations of the two methods. The black line is the true cardinality of the targets, and the magenta dotted line and the blue dotted line represent estimated standard deviations of the ET-MSMeMBer filter and the proposed filter, respectively. We can observe that compared with the ET-MS-MeMBer filter, the proposed method obtains a smaller standard deviation of the estimated cardinality. Figure 6 shows the OSPA errors of the two methods. Note that, for both two methods, the delay in response to target birth and target death leads to the high peaks at the corresponding times. Nevertheless, the OSPA error of the proposed method is still smaller than that of the ET-MS-MeMBer filter.

We also consider the case of a varying number of sensors with $p_{i, d}=0.7$ and $\lambda_{i}=80$ for each sensor $i$. As before, 50 Monte Carlo runs are performed. Figure 7 shows the time-

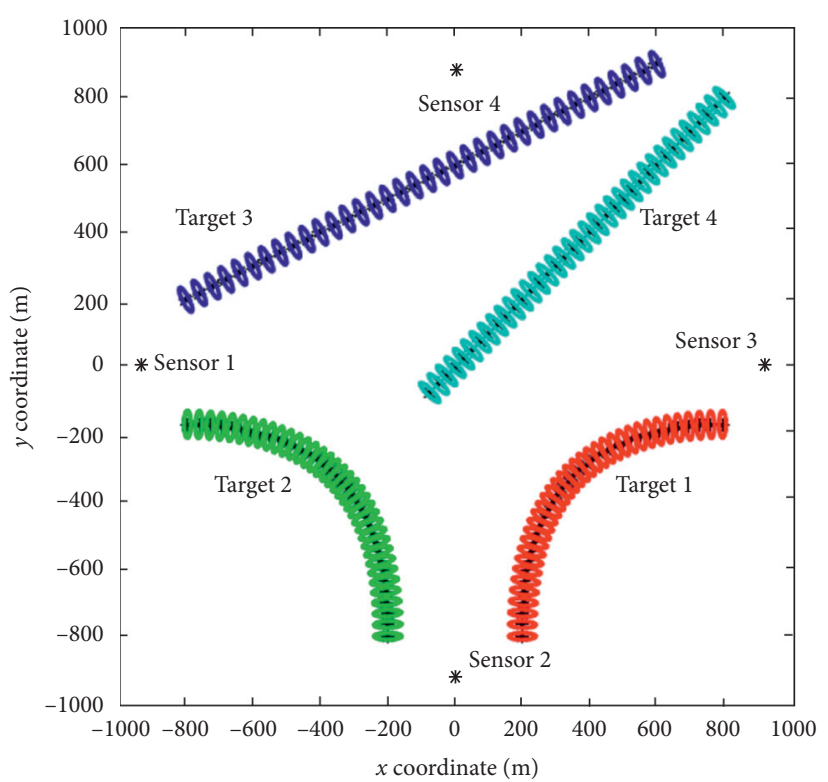

FIGURE 1: Target trajectories.

TABLE 1: The true movement of targets.

\begin{tabular}{lc}
\hline The initial position of targets & Survival time \\
\hline$\left[200 \mathrm{~m},-800 \mathrm{~m}, 0 \mathrm{~m} / \mathrm{s}, 35 \mathrm{~m} / \mathrm{s}, 1 \mathrm{~m} / \mathrm{s}^{2},-1 \mathrm{~m} / \mathrm{s}^{2}\right]$ & $(5 \mathrm{~s} \sim 40 \mathrm{~s})$ \\
{$\left[-200 \mathrm{~m},-800 \mathrm{~m}, 0 \mathrm{~m} / \mathrm{s}, 35 \mathrm{~m} / \mathrm{s},-1 \mathrm{~m} / \mathrm{s}^{2},-1 \mathrm{~m} / \mathrm{s}^{2}\right]$} & $(45 \mathrm{~s} \sim 80 \mathrm{~s})$ \\
{$\left[-800 \mathrm{~m}, 200 \mathrm{~m}, 40 \mathrm{~m} / \mathrm{s}, 20 \mathrm{~m} / \mathrm{s}, 0 \mathrm{~m} / \mathrm{s}^{2}, 0 \mathrm{~m} / \mathrm{s}^{2}\right]$} & $(5 \mathrm{~s} \sim 40 \mathrm{~s})$ \\
{$\left[800 \mathrm{~m}, 800 \mathrm{~m},-25 \mathrm{~m} / \mathrm{s},-25 \mathrm{~m} / \mathrm{s}, 0 \mathrm{~m} / \mathrm{s}^{2}, 0 \mathrm{~m} / \mathrm{s}^{2}\right]$} & $(45 \mathrm{~s} \sim 80 \mathrm{~s})$ \\
\hline
\end{tabular}

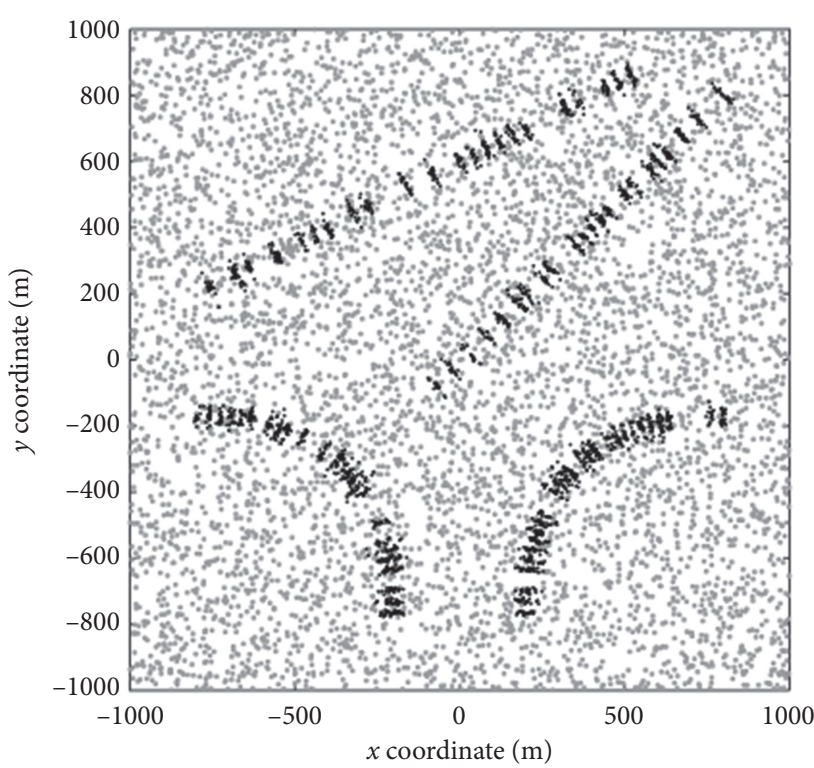

Figure 2: The measurements of sensor 1. The black dot represents the target measurement, and the gray dot represents the clutter.

averaged OSPA error of the two methods, and Figure 8 shows the running time. We observe that as the number of the sensors increases, the time-average OSPA error of both two methods decreases, however, at the cost of the growing 


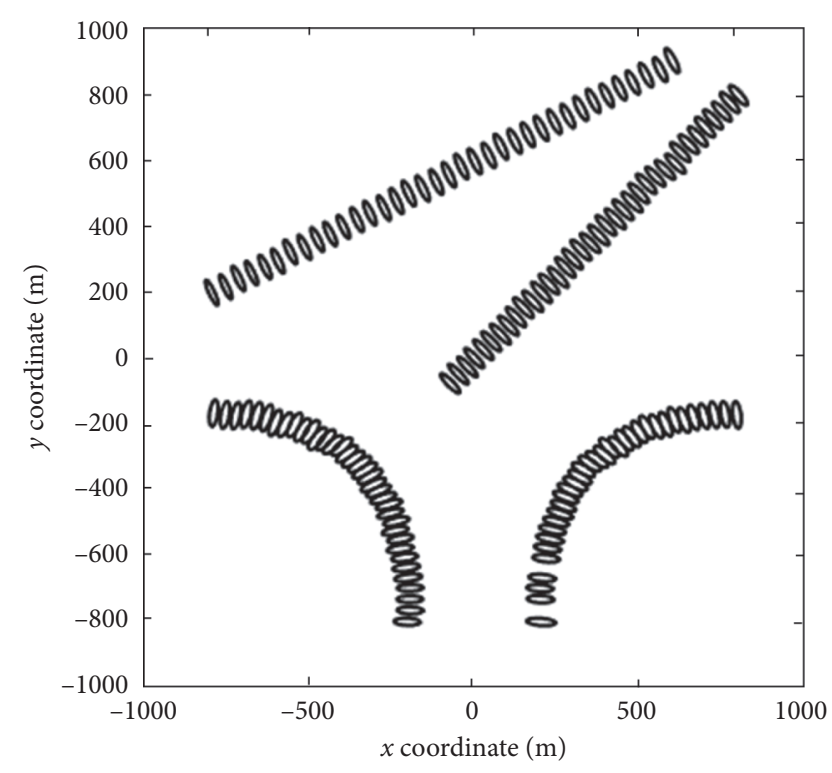

FIgURE 3: The estimated states of the proposed method.

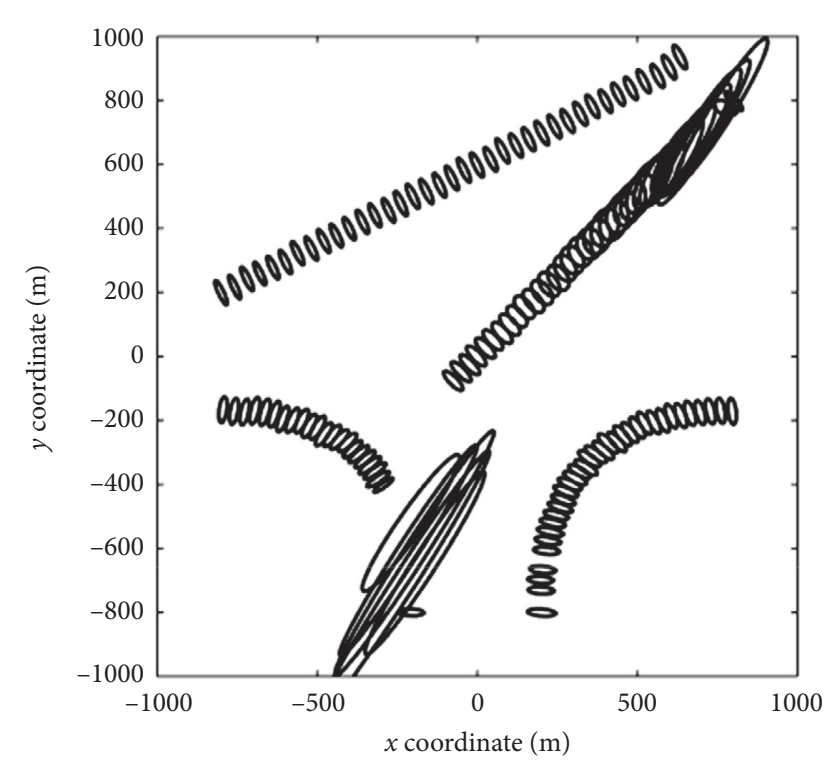

FIgUre 4: The estimated states of the ET-MS-MeMBer filter.

computational time. Comparatively, the proposed method performs better.

Figure 9 shows the time-averaged OSPA error of the two methods in scenarios with different clutter intensities. The corresponding running time of the two methods is shown in Figure 10 . The clutter density $\lambda_{i}$ is varied in a range of $\{5,20$, $40,60,80,100\}$. Besides, the number of sensors is 3 , and the



Figure 5: Comparison of the estimated cardinality.

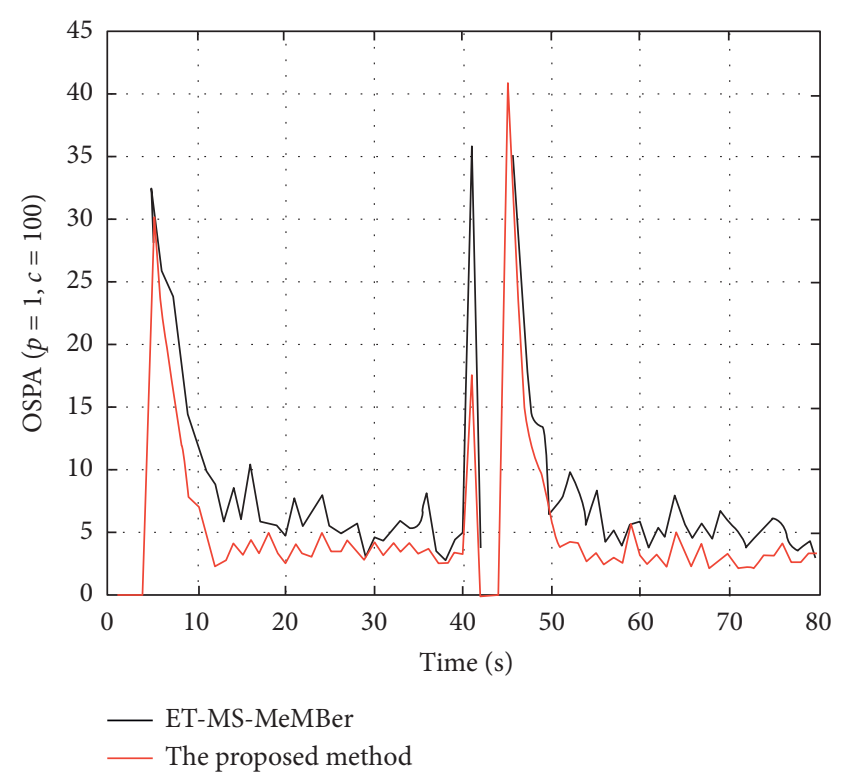

FIgure 6: Comparison of the OSPA error.

detection probability is $p_{i, d}=0.7$. It is observed that the OSPA error and running time of the two methods are similar in the low clutter density case, i.e., $\lambda_{i}=5$. With the increase of the clutter intensity, the filtering performance of the two methods decreases. However, the proposed method requires less time and has smaller OSPA errors when the clutter intensity is high. 


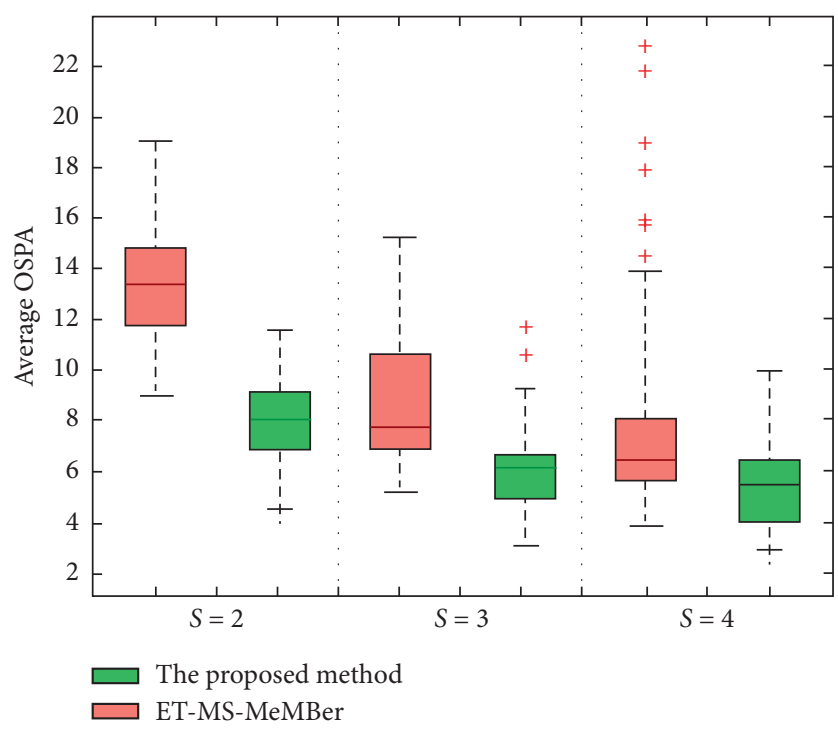

Figure 7: Time-average OSPA errors with different sensor numbers.



FIgURE 8: Running time with different sensor numbers.

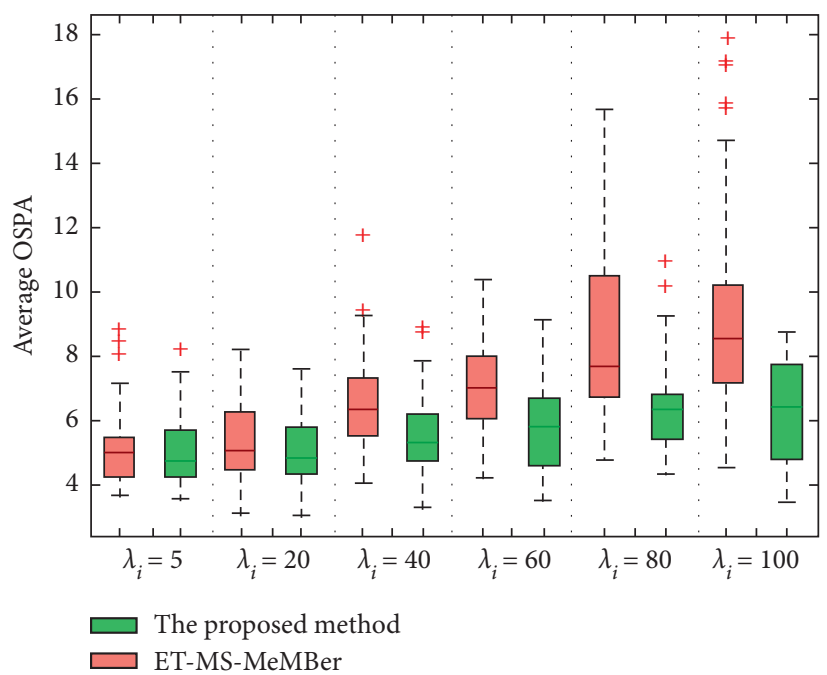

Figure 9: Time-average OSPA errors with different clutter intensities. 


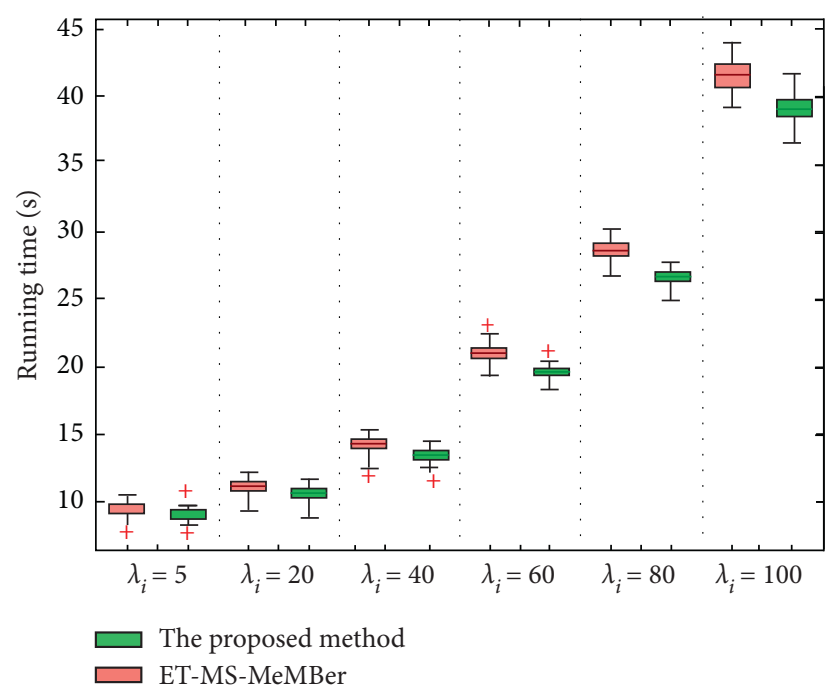

FIgURE 10: Running time with different clutter intensities.

\section{Conclusions}

In this paper, we propose a robust MS-MeMBer filter for extended-target tracking in clutters. We modify the multisensor measurement partitioning process by considering the constraint of the clutter measurement set to make it robust under conditions with different clutter intensities. Accordingly, we propose the single-target multisensor likelihood function for the clutter case. Simulation results show that compared with the ET-MS-MeMBer filter, the proposed method has smaller OSPA errors with less time consumption under high-clutter-density conditions.

\section{Data Availability}

The simulation data used to support the findings of this study are included within the article. No measured data were used to support this study.

\section{Conflicts of Interest}

The authors declare that they have no conflicts of interest.

\section{References}

[1] D. Reid, "An algorithm for tracking multiple targets," IEEE Transactions on Automatic Control, vol. 24, no. 6, pp. 843854, 1979.

[2] Z. W. Wang, J. P. Sun, Q. Li, and G. H. Ding, "A new multiple hypothesis tracker integrated with detection processing," Sensors, vol. 19, no. 23, Article ID 5278, 2019.

[3] G. Karl and M. Baum, "Extended object tracking: introduction, overview and applications," Journal of Advances in Information Fusion, vol. 12, pp. 139-174, 2017.

[4] R. Mahler, Statistical Multisource-Multitarget Information Fusion, Artech House, Norwood, MA, USA, 2007.

[5] F. Perea and H. W. De Waard, "Greedy and K-greedy algorithms for multidimensional data association," IEEE Transactions on Aerospace and Electronic Systems, vol. 47, no. 3, pp. 1915-1925, 2011.
[6] N. Chenouard, I. Bloch, and J. C. Olivo-Marin, "Multiple hypothesis tracking in microscopy images," in Proceedings of the IEEE International Symposium on Biomedical Imaging: From Nano to Macro, pp. 1346-1349, Boston, MA, USA, July 2009.

[7] S. Schwoegler, J. Holsopple, and S. Blackman, "On the application of multiple hypothesis tracking to the cyber domain," in Proceedings of the IEEE 2011 International Conference on Information Fusion, pp. 1-6, Chicago, IL, USA, July 2011.

[8] B. Rakdham, M. Tummala, and P. E. Pace, "Boost phase ballistic missile defense using multiple hypothesis tracking," in Proceedings of the IEEE 2007 International Conference on System of Systems Engineering, pp. 1-6, San Antonio, TX, USA, April 2007.

[9] R. Mahler, "Multitarget Bayes filtering via first-order multitarget moments," IEEE Transactions on Aerospace Electronic System, vol. 39, pp. 1152-1178, 2004.

[10] K. Da, T. Li, Y. Zhu, H. Fan, and Q. Fu, "Recent advances in multisensor multitarget tracking using random finite set," Frontiers of Information Technology \& Electronic Engineering, vol. 22, no. 1, pp. 5-24, 2021.

[11] B.-N. Vo and W.-K. Ma, "The Gaussian mixture probability hypothesis density filter," IEEE Transactions on Signal Processing, vol. 54, no. 11, pp. 4091-4104, 2006.

[12] B.-T. Vo, B.-N. Vo, and A. Cantoni, "Analytic implementations of the cardinalized probability hypothesis density filter," IEEE Transactions on Signal Processing, vol. 55, no. 7, pp. 3553-3567, 2007.

[13] T. Li, X. Wang, Y. Liang, and Q. Pan, "On arithmetic average fusion and its application for distributed multi-Bernoulli multitarget tracking," IEEE Transactions on Signal Processing, vol. 68 , pp. 2883-2896, 2020.

[14] A.-A. Saucan, M. J. Coates, and M. Rabbat, "A multisensor multi-Bernoulli filter," IEEE Transactions on Signal Processing, vol. 65, no. 20, pp. 5495-5509, 2017.

[15] R. Mahler, "PHD filters for nonstandard targets, I: extended targets," in Proceeding of the 12th International Conference on Information Fusion, pp. 915-921, Seattle, WA, USA, July 2009.

[16] K. Granstrom, C. Lundquist, and O. Orguner, "Extended target tracking using a Gaussian-mixture PHD filter," IEEE 
Transactions on Aerospace and Electronic Systems, vol. 48, no. 4, pp. 3268-3286, 2012.

[17] K. Granstrom and U. Orguner, "A PHD filter for tracking multiple extended targets using random matrices," IEEE Transactions on Signal Processing, vol. 60, no. 11, pp. 56575671, 2012.

[18] K. Granstrom, A. Natale, P. Braca, G. Ludeno, and F. Serafino, "Gamma Gaussian inverse Wishart probability hypothesis density for extended target tracking using X-band marine radar data," IEEE Transactions on Geoscience and Remote Sensing, vol. 53, no. 12, pp. 6617-6631, 2015.

[19] U. Orguner, C. Lundquist, and K. Granstrom, "Extended target tracking with a cardinalized probability hypothesis density filter," in Proceeding of the 14th International Conference on Information Fusion, pp. 1-8, Chicago, IL, USA, July 2011.

[20] M. Beard, S. Reuter, K. Granstrom, B.-T. Vo, B.-N. Vo, and A. Scheel, "Multiple extended target tracking with labeled random finite sets," IEEE Transactions on Signal Processing, vol. 64, no. 7, pp. 1638-1653, 2016.

[21] K. Granstrom, M. Fatemi, and L. Svensson, "Poisson multiBernoulli mixture conjugate prior for multiple extended target filtering," IEEE Transactions on Aerospace and Electronic Systems, vol. 56, no. 1, pp. 208-225, 2020.

[22] K. Granstrom, M. Fatemi, and L. Svensson, "Gamma Gaussian inverse-Wishart Poisson multi-Bernoulli filter for extended target tracking," in Proceeding of the 19th International Conference on Information Fusion, pp. 893-900, Heidelberg, Germany, July 2016.

[23] Y. Xia, K. Granstrom, L. Svensson, A. F. García-Fernández, and J. L. Williams, "Extended target Poisson multi-Bernoulli mixture trackers based on sets of trajectories," in Proceeding of the 22th International Conference on Information Fusion, pp. 1-8, Ottawa, ON, Canada, July 2019.

[24] Z. Zhang, J. Sun, Q. Li, C. Liu, and G. Ding, "A novel MSMeMBer filter for extended targets tracking," IEEE Access, vol. 8, pp. 37596-37607, 2020.

[25] K. Gilholm, S. Godsill, and S. Maskell, "Poisson models for extended target and group tracking," in Proceedings of the Signal and Data Processing of Small Targets, pp. 1-12, San Diego, CA, USA, September 2005.

[26] D. Schuhmacher, B.-T. Vo, and B.-N. Vo, "A consistent metric for performance evaluation of multi-object filters," IEEE Transactions on Signal Processing, vol. 56, no. 8, pp. 34473457, 2008. 